\title{
On Phonetically Based Phonology
}

\author{
Marc van Oostendorp
}

September 14, 2005

Bruce Hayes, Robert Kirchner and Donca Steriade (eds.) Phonetically Based Phonology. Cambridge: Cambridge University

Press, 2004. A website accompanies the book: http://www.linguistics.ucla.edu/people/hayes/PBP/

"The entire discussion of phonology in this book suffers from a fundamental theoretical inadequacy." "This is the first sentence of the famous Chapter Nine of Chomsky \& Halle (1968). "The problem is", the authors explain, "that our approach to features, to rules, and to evaluation has been overly formal", and "[i]n particular, we have not made any use of the fact that the features have intrinsic content."

Certain things may have changed in phonological theory since these words were written, but the problem of Chomsky \& Halle (1968) is still awaiting a fully satisfactory solution. As a matter of fact, we may observe that at present the assumptions on this issue converge wildly among researchers, even those purportedly subscribing to the same framework, Optimality Theory. This very strong variation of opinions on issues which seem so fundamental to our field may be seen as somewhat discomforting - it is hard to speak of progress if we have not even been able to agree on the basics in the past 35 years - but of course it also makes for interesting debates.

The book under review here, Hayes et al. (2004), gives an overview of work done (mostly at the University of California, Los Angeles) to solve the Chapter Nine problem within one very specific set of assumptions, combining recent insights into phonetics with phonological Optimality Theory. Except for one article, by Blevins \& Garrett, all papers in this book assume that human beings - in particular, languageacquiring children — have some amount of implicit knowledge about phonetics, and this assumption renders the existence of much abstract phonology superfluous. The articles discuss various implications of this stance for our views on classical problems

\footnotetext{
${ }^{1}$ Thanks are due to Ben Hermans, Frans Hinskens and Nina Topintzi for comments. All disclaimers
} apply. 
in phonological theory such as sonority (Wright), place assimilation (Jun), vowel harmony (Kaun), contour tones (Zhang), vowel reduction (Crosswhite), contrast (Flemming), syllable weight (Gordon), lenition (Kirchner) and the OCP (Frisch). The introduction to the book by Hayes and Steriade presents a clear and lucid overview of the basic philosophy behind the enterprise of phonetically based phonology (henceforth PBP).

I consider Phonetically Based Phonology a very important book, because it represents in an intelligent and coherent way a variety of work within one given theoretical framework. In order for the field of phonology to not fall apart in many different pieces, I believe it is important that there is a serious debate about the differences in assumptions. As such, the present book is important because it represents one point of view; but unfortunately it does not pay a lot of attention to alternatives.

In this review, I will not go into each of the articles in detail. The variety of topics discussed in this volume is too large to even make it possible to do justice to every contribution - and the reader who wants to have an overview of the various topics that are discussed may consult the website of the first editor of this book. Instead of this, I want to go into two topics which seem particularly important for the evaluation of the framework of phonetically based phonology as a whole: the comparison to classical assumptions within Optimality Theory, and the relation to other models of phonology-phonetics interaction.

\section{Differences with classical Optimality Theory}

The central difference between PBP and classical OT is the source of the constraint set. Whereas Prince \& Smolensky (1993) assumed that all constraints are universal and innate, PBP subscribes to neither of those assumptions. In contrast, it is assumed that the constraints are derived by speakers or learners on the basis of the available evidence, and of implicit phonetic knowledge, "the speakers' partial understanding of the physical conditions under which speech is produced and perceived" (Hayes and Steriade, p. 1). This knowledge is potentially universal - every human being can have access to it. But it is not (necessarily) completely innate. Although none of the articles is very explicit on this, and the term acquisition is regrettably absent from the index, it is assumed that "Universal Grammar (UG) [is] primarily [...] a set of abstract analytical predispositions that allow learners to induce grammars from the raw facts of speech" (cf. Hayes, 1999).

One important reason for taking this step is that it is believed by the proponents of PBP that the cognitive universals in UG are inherently arbitrary, and that we should have as little of them as possible. This is in itself nothing but an application of Occam's Razor, but it should be borne in mind that the phonetic knowledge that is 
posited by the scholars working in PBP will also come with a substantial cognitive cost: an empty UG does not come for free if we burden the minds of our speakers at the same time with pieces of knowledge such as the following: "[Certain putatively universal constraint rankings] reflect the speaker's knowledge that [...] a syllable should be able to host a tone with a lower complexity before it can host a tone with higher complexity" (Zhang, p. 159). It is hard to evaluate objectively what the relative costs and merits of innate constraints vs. the innate capacity to do acute phonetic observations are. It therefore seems a little bit strong to suppose that PBP represents the "null hypothesis" in any reasonable sense of that term.

Another relatively new development of PBP compared to classical OT and other earlier generative approaches is the stronger attention paid to perceptual factors. Earlier approaches to the phonetics-phonology interface, such as Chapter Nine of SPE, concentrated almost exclusively to articulatory phonetics. In this volume, almost all articles have a perceptual component to them. This is even true for instance for Jun's article on place assimilation, or Kaun's article about vowel harmony: two phenomena which on first sight seem perfectly amenable to an articulatory analysis. Kaun's very interesting contribution, in particular, shows how an understanding of perception can put many aspects of the typology of (Turkic) vowel harmony into a new perspective. For instance, the fact that high vowels are often good targets for harmony, but function as triggers less often, is attributed to the fact that rounding differences are particularly easy to perceive on high vowels. This follows immediately from the proposition that vocalic properties spread from positions where they stand a lesser chance of being detected to positions where they may be perceived more easily.

One might consider the attention to perceptual factors a positive development, since there is no a priori reason to restrict our interest in phonetics to articulation. Furthermore, it is obviously true that if there are two independent explanations of one and the same phenomenon - for instance, one phonetic explanation and one phonological explanation - scientists should not be very satisfied, and one explanation should be dispensed with. Reduction of phonological explanations to phonetic ones is one option, so for those who are convinced that such a reduction is viable, there is every reason to build up a complete theory of phonetics.

On the other hand, this type of reasoning does not lead us to posit severe restrictions on our model, in particular since it is usually considered to be the case that the interests of articulation are different from, or even conflicting with, those of perception. It is hard to think of states of affairs which could not have some 'explanation' in terms of either articulation or perception.

Consider for instance the discussion of the minimal vowel set /i u a/ by Crosswhite (p. 195-196). It turns out that several phonetic reasons can - and have been given for the special status of these three vowels: (i) "the corner vowels /i, $u$, $\mathrm{a}$ / are special in that they are maximally acoustically distinct", (ii) "the vowels /i, u, 
a/ are also special in terms of their production: they all show quantal effects", (iii) "the vowels /i, u, a/ are distinguished by having not only articulatory stability, but also by the fact that they do not share this characteristic with adjacent qualities", (iv) "the quantal vowels /i, u, a/ and /y/ are perceptually special by virtue of having spectral prominences caused by convergences: either proximity of two formants $[\ldots]$ or proximity of the first formant and the fundamental frequency [...]. From this, Crosswhite concludes that the status of the corner vowels /i, u, a/ "does not seem to be linked to any single characteristic, but perhaps to the amalgamation of several". However, it is not particularly convincing if more than one explanation can be given for the same factum; this doubling of explanations is an important reason to try to reduce phonology to phonetics in the first place. Further, it would need to be shown that we cannot give common characteristics of other vowel sets (Crosswhite discusses the vowel set /i, $\mathrm{u}, \mathrm{J} /$ which shares at least some of these characteristics, but does not seem to play a special role in language typology. It remains somewhat mysterious why such would be the case.)

Another example of how reference to phonetics does not necessarily lead to a theory which is more precise, is Jun's analysis of place assimilation (pp. 58-86), which is based on two constraint schemas:

(1) a. PRESERVE ${ }_{n}$ (place): Preserve at least $n$ per cent of the perceptual cues for place of articulation.

b. WEAKENING $m$ : Do not produce an articulatory gesture whose effort cost is at least $m$.

The definitions for both constraints are not self-evident, since there is no a priori way of defining either how much 'per cent' of perceptual cues are preserved in some input-output pair, nor is there a well-established way of measuring effort costs. The reasoning therefore has to be based on rather general considerations, and probably the relevant measurements can be done in many different ways, which would give different outcomes, and therefore different scales and different predictions.

Somewhat confusing is also the inclusion of an article by Stefan Frisch in this volume ("Language processing and segmental OCP effects", pp. 346-371) which seems to be based on psycholinguistic work on processing rather than on articulation and perception; this raises the question how exactly we define 'phonetics'.

To this point, is interesting to note that Hayes and Steriade distinguish between 'inductive' and 'deductive' approaches to the theory of markedness:

Most attempts to discover markedness principles in phonology have proceeded, until recently, in inductive fashion: phonologists accumulate factual observations about languages and, in due course, a cluster of such 
observations coheres into a law. [...] [I]n most cases, the laws originate as generalisations over known languages, not as principles explaining why these laws should be expected to hold. A set of such laws, when they survive peer review, forms a proposed theory of markedness.

$[\ldots]$ The work reported in this volume proceeds deductively $[\ldots]$ by asking at the outset variants of the following questions: Are there general properties distinguishing marked from unmarked phonological structures, and, if so, what are they?

[...] Deductive research on phonological markedness starts from the assumption that markedness laws obtain across languages not because they reflect structural properties of the language faculty, irreducible to non-linguistic factors, but rather because they stem from speakers' shared knowledge of the factors that affect speech communication by impeding articulation, perception, or lexical access.

It seems a little bit too strong to equate the distinction between earlier approaches and phonetically based phonology with one between inductive and deductive research.

Almost all concrete works of research combine both aspects in one way or another. In the best generative work on markedness (I suppose the authors are referring here to other generative traditions, including more formalist versions of OT), one indeed generalises over known languages, but one does this in a theoretically inspired way: the generalisations take the form of a model of segmental, syllabic or metrical organisation. This model is then taken as a set of axioms from which new hypotheses are deduced. Inversely, phonetically inspired work can hardly live on deductive principles alone; in almost every paper in the present book, the author tries to understand some (inductively established) generalisation, and to find an articulatory or perceptual explanation for it, in a post hoc fashion.

As a matter of fact, one could wonder how 'inductive' the approach of PBP really is. In order to be inductive, we need to have a set of axioms from which hypotheses can be derived and it is not clear that such a set of axioms is available within phonetics; in any case, no such set is presented in any of the contributions to this book. To the contrary, phonetics is used at various places in this book as a tool to derive something which has been established beforehand; for example, Wright's article provides a long list of perceptual cues and factors for cue robustness in order to derive the effects of Sonority Sequencing, which is a classical principle of generative phonology, which has been arrived at through 'induction'. The deduction of this fact from phonetic factors is therefore ad hoc at best. I do not wish to argue that this is a bad thing; I think it is an inevitable aspect of any scientific enterprise. But to claim then that PBP is particularly deductive, with the implicit suggestion that deduction is superior to induction, is not very helpful. 
A final point to note in the comparison of PBP to other generative theories is on the relation between formalism and substance. This was the issue of the remark at the beginning of Chapter Nine of SPE, and it is brought up again in this book at various places. One particularly relevant contribution in this respect is the article by Gordon (p. 277-312), who gives an overview of various phonetic factors that may contribute to the weight of a syllable, such as length, vocalic height and sonorancy of coda consonants, but also shows that in actual phonological systems there seems to be an "upper limit of formal complexity tolerated by weight distinctions" and states (p. 289-290): "I thus offer the following definition of complexity as a working hypothesis: a weight distinction is too complex if it refers to more than one place predicate." This notion of complexity is purely formal and therefore purely phonological in Gordon's view: even though there is no articulatory or perceptual reason to avoid this type of complexity, the universal grammar may impose those restrictions - notice by the way that again this follows from induction and not from anything else.

\section{Other models of phonology-phonetics interaction}

The relation between phonology and phonetics has been the object of many studies, and too little debate, in recent years; most of the contributions in Phonetically Based Phonology represent just one line of thought. Even authors who seem to pursue a similar program but in a different geographic location are not included; Morrison (2005) mentions Paul Boersma in a review of this book, and this is a name I would have liked to see included as well, if only because his views on the relation between perception and articulation in the grammar seem to be slightly different from those of the authors presented in this book. Roughly, in Boersma's model (Boersma, 1998, and much subsequent work) there are separate grammars for perception and articulation, while most authors in PBP seem to assume that there is one grammar where both types of constraints are interspersed.

But even more regrettable is the fact that the authors in this volume do not pay a lot of attention to other models of phonology-phonetics interaction in their own writings either. In the review already referred to, Morrison (2005) mentions the work of Björn Lindblom and John Ohala, and others could have been discussed as well e.g. the work of Janet Pierrehumbert and Joan Bybee. I also wish to point out that there have always been voices in the debate defending opposite views, but in most cases, reference to them is missing in this book. For instance, McCarthy (2002) cites several problems with Edward Flemming's particular implementation of PBP; none of them are acknowledged in Flemming's present paper. Similarly, Mark Hale and Charles Reiss of Concordia University have been criticizing 'substantive' approaches to phonology for a number of years (see for instance Hale \& Reiss, 2000); as far as 
I can see, none of their publications is cited. ${ }^{2}$ The issue is not whether or not these criticisms are right; the issue is that there can be no debate if there are no crossreferences to other traditions. The field then runs the danger of falling apart into a number of schools, each concerned with its own problems and its own methodology.

It has to be mentioned that one article is an exception in this volume: Blevins and Garret present a view which is very different from that of all other contributors ("The evolution of metathesis", p. 117-156). These authors offer a four-way classification of phonological theories: synchronic and nonfunctionalist (e.g. classical OT), synchronic and functionalist (e.g. PBP), diachronic and functionalist (e.g. Grammont, 1950), diachronic and nonfunctionalist. The work of Blevins and Garret falls within the last category - and thus is the reverse of the rest of PBP. In their view, speakers do not need to have any kind of phonetic knowledge. Languages change because speakers (in particular, language learning children) interpret the phonetic signal they hear in a different way. Phonetically natural misapprehensions are much more likely to occur than unnatural ones, and this is the way in which natural patterns will tend to creep into the linguistic structure. There is no real need for an independent phonetic markedness component in the grammar any more: phonetics is external to the speakers' heads. (This view has been extensively defended in Blevins, 2004)

The editors of the volume explain in their introduction what their objections against this approach are - mainly, that they believe that certain language changes seem to be teleological, i.e. moving in the direction of a 'less marked' structure. They conclude by saying that they "believe that most of the evidence that could bear on either side's position remains to be gathered or considered, and thus that further attention to this debate could lead to research progress."

It remains therefore a regrettable fact that the authors have not payed more attention to differences between their own school of thought and other schools, past or present. For instance, with respect to the 'teleology' we could wonder what 'goal' the language system is trying to achieve. Much here depends on our conception of the 'function' of language. Underlying almost all work within PBP is the conception that the primary - if not the only - function of language is to transmit information: I have a concept in my head, I speak and if the speech act has been succesful you will have the same concept in your head. Seen from this perspective, it is thus important for the speaker to make her signal as clear as possible without taking too much effort.

No argument is given, however, for the assumption that 'efficient communication' is the central - maybe the only relevant - function of language, and there is no discussion of any of the other functions human language may have. Since Jakobson (1960) it is usually assumed the following functions can be assigned to human

\footnotetext{
${ }^{2}$ There is some discussion of Anderson (1981) who defended roughly the same position as Hale and Reiss — in the early 1980s.
} 
language:

1. emotive function, expressing the attitudes or moods of the speaker

2. conative function, directing the listener towards some action, a change of attitude, etc.

3. referential function, transferring information from one head to the other

4. phatic function, keeping the line of contact open between speaker and listener

5. poetic function, exploring the properties of language (rhythm, syntactic structures)

6. metalingual function, discussing the features of other utterances

It seems clear that some of these functions are more 'important' than others; in particular, that the metalingual function is somehow parasitic on other functions - it is unlikely that somebody would come up with a theory which would establish that the metalingual function is the most important one. However, it is not a priori clear what the central function is, and it is a little bit discomforting that arguments as to why the referential function should be seen as the central one rather than any of the others. ${ }^{3}$

But also given the implicit assumptions of PBP, it is not always clear what the function of individual objects proposed in the model is. One case is Dispersion Theory, presented in a very clear and lucid way by Flemming in this book (pp. 232-276). Within Dispersion Theory it is assumed that vowels are part of a vowel system, and these vowel systems are organized in such a way that vowels are maximally different from each other. One interesting consequence of this is that these theories incorporate certain insights of structuralism which were not available in earlier stages of generative grammar (Scheer, 2004). One of the central insights of Saussure of course was that dans la langue, il n'y a que des différences. Every element of a linguistic system only exists by virtue of the fact that it is in opposition to other elements; indivudual vowels, for instance, exist by virtue of their being different. One of the claims of Dispersion Theory is that the phonetic distance corresponding to these dimensions are 'optimised' in OT tableaux.

It seems to me that it follows from this that something like a 'vowel system' is a concrete object in the theory. Since PBP subscribes to the generative idea that the grammar is a mental object, it follows from this that we have something like a vowel system in our mind, independent of the concrete words in the lexicon. It does not become clear, however, what the functional need for such an object is: why would the language user come equipped with a mental map of a vowel triangle on which

\footnotetext{
${ }^{3}$ See Hale \& Reiss (2000) for a similar criticism; these authors point out that if we would assume that it is the function of the language system to confuse the listener and exhaust the speaker, we may still set up a constraint system which does exactly the same work as what is done in functional work. For a critical evaluation of functional grounding in syntactic OT see Newmeyer (2002), as well as the reply by Bresnan \& Aissen (2002) following it.
} 
Dispersion works and where the vowels in individual words are somehow related to the vowels in the triangle? In the standard generative view, there is no such thing as a vowel system: it is an epiphenomenon at best. From a functional point of view, it would make more sense to disperse words than individual sounds, as McCarthy (2002) points out. Vowel systems are abstract objects for which there is no obvious functional need; issues such as these would have warranted more discussion.

\section{Conclusion}

The issue of how phonetics and phonology are related is a very important one, and it is potentially very benificial for both fields of study to consider their interaction more closely. This is one of the goals of PBP, which furthermore sets out one specific proposal and applies it in a more or less coherent way to a number of phenomena which can be considered to be crucial for any phonological model. Many of the assumptions underlying work in PBP are not shared by other phonologists; it would therefore have been helpful, first, if these assumptions would have been laid out more explicitly, and, second, if they would have been compared more carefully to other possible sets of assumptions. Phonology is a field which already has some tradition; it is not helpful to ignore this tradition, or dismiss it as mere inductivism. But this of course can also be seen as a programme for future work, both for the authors and for the readers of this thought-provoking volume.

\section{Bibliography}

Anderson, Stephen (1981). 'Why phonology isn't 'natural'. Linguistic Inquiry, 12: 493-539.

Blevins, Juliette (2004). Evolutionary phonology. The emergence of sound patterns. Cambridge: Cambridge University Press.

Boersma, Paul (1998). Functional Phonology. Ph.D. thesis, University of Amsterdam.

Bresnan, Joan \& Judith Aissen (2002). 'Optimality and functionality: Objections and refutations'. Natural Language and Linguistic Theory, 20, 1: 81-95. URL http://www-lfg.stanford.edu/bresnan/fot-final.pdf.

Chomsky, Noam \& Morris Halle (1968). The Sound Pattern of English. Cambridge, MA: The MIT Press.

Grammont, Maurice (1950). Traité de phonétique. Parus: Delagrave, 4 edn.

Hale, Mark \& Charles Reiss (2000). 'Phonology as cognition'. In: Burton-Roberts, N., Philip Carr, \& Gerard Docherty (eds.), Phonological Knowledge: Conceptual and Empirical Foundations, pp. 161-184. Oxford: Oxford University Press. 
Hayes, Bruce (1999). 'Phonetically-driven phonology: the role of Optimality Theory and grounding'. In: Darnell, Michael, Edith Moravscik, Michael Noonan, Frederick Newmeyer, \& Kathleen Wheatly (eds.), Functionalism and Formalism in Linguistics, Volume I: General Papers, pp. 243-285. Amsterdam: John Benjamins. URL http://www.linguistics.ucla.edu/people/hayes/ phonet.htm.

Hayes, Bruce, Robert Kirchner, \& Donca Steriade (eds.) (2004). Phonetically Based Phonology. Cambridge: Cambridge University Press. URL http: //www.linguistics.ucla.edu/people/hayes/PBP/

Jakobson, Roman (1960). 'Linguistics and Poetics: Closing Statement'. In: Sebeok, Thomas (ed.), Style in Language. Cambridge, MA: Massachusetts Institute of Technology.

McCarthy, John (2002). A Thematic Guide to Optimality Theory. Research Surveys in Linguistics. Cambridge: Cambridge University Press.

Morrison, George (2005). 'Review of Hayes et al. (2004)'. Linguist List, 16, 1400. URL/http://www.ling.ed.ac.uk/linguist/issues/16/ 16-1400.html

Newmeyer, Frederick (2002). 'Optimality and functionality: A critique of functionally-based optimality-theoretic syntax'. Natural Language and Linguistic Theory, 20, 1: 43-80. URL http://faculty.washington.edu/fjn/ Newmeyer_OT_NLLT.pdf.

Prince, Alan \& Paul Smolensky (1993). 'Optimality Theory: Constraint Interaction in Generative Grammar'. Manuscript, Rutgers University and University of Colorado at Boulder. Available at ROA.

Scheer, Tobias (2004). 'Présentation du volume. En quoi la phonologie est vraiment différente'. Corpus, 3: 5-84. 